\title{
Equivalent Single-Junction Model of Superconducting Quantum Interference Devices in the Presence of Time-Varying Fields
}

\author{
R. De Luca \\ Department of Physics “E. R. Caianiello," University of Salerno, 84084 Fisciano (SA), Italy \\ Correspondence should be addressed to R. De Luca, rdeluca@unisa.it
}

Received 28 July 2011; Accepted 13 September 2011

Academic Editors: N. Hatakenaka, F. Marsiglio, and T. Schurig

Copyright ( $) 2011$ R. De Luca. This is an open access article distributed under the Creative Commons Attribution License, which permits unrestricted use, distribution, and reproduction in any medium, provided the original work is properly cited.

The reduced dynamical model of a two-junction quantum interference device is generalized to the case of time-varying externally applied fluxes with a d. c. component and an oscillating addendum whose frequency is comparable with the inverse of the characteristic time for flux dynamics within the superconducting system. From the resulting effective single-junction model for null inductance of the superconducting loop, it can be seen that the critical current of the device shows a dependence on the frequency and amplitude of the oscillating part of the applied flux. It can therefore be argued that the latter quantities can be considered as control parameters in the voltage versus applied flux curves of superconducting quantum interference devices.

\section{Introduction}

It is well known that the electrodynamic properties of SQUIDs (superconducting quantum interference devices) can be obtained by means of the dynamics of the Josephson junction(s) in the system [1-3]. Due to the intrinsic macroscopic coherence of superconductors, r. f. SQUIDs have been proposed as basic units (qubits) in quantum computing [4]. In the realm of quantum computing, nondissipative quantum systems with small (or null) inductance parameter and finite capacitance of the Josephson junctions (JJs) are usually considered [5]. The mesoscopic nonsimply connected classical devices, on the other hand, are generally operated and studied in the overdamped limit with negligible capacitance of the JJs and small (or null) values of the inductance parameter. Nonetheless, the latter find application in a large variety of fields, from biomedicine to aircraft maintenance [3], justifying actual scientific interest in them.

As far as d. c. SQUIDs are concerned, these systems can be analytically described by means of a single-junction model [6]. The elementary version of the single-junction model for a d. c. SQUID takes the inductance $L$ of a single branch of the device to be negligible, so that $\beta=L I_{J} / \Phi_{0} \approx 0$, where $\Phi_{0}$ is the elementary flux quantum and $I_{J}$ is the average value of the maximum Josephson currents of the junctions. In this way, the Josephson junction dynamics is described by means of a nonlinear first-order ordinary differential equation (ODE) written in terms of the phase variable $\phi$, which is the average of the gauge-invariant superconducting phase differences, $\phi_{1}$ and $\phi_{2}$, across the two junctions in the SQUID. By considering a device with equal Josephsons junction in each of the two symmetric branches, the dynamical equation of the variable $\phi$ can be written as follows [1]:

$$
\frac{d \phi}{d \tau}+(-1)^{n} \cos \pi \psi_{\mathrm{ex}} \sin \phi=\frac{i_{B}}{2},
$$

where $n$ is an integer denoting the number of fluxons initially trapped in the superconducting interference loop, $\tau=2 \pi R I_{J} t / \Phi_{0}=t / t_{J}, R$ being the intrinsic resistive junction parameter, $\psi_{\mathrm{ex}}=\Phi_{\mathrm{ex}} / \Phi_{0}$ is the externally applied flux normalized to $\Phi_{0}$, and $i_{B}=I_{B} / I_{J}$ is the bias current normalized to $I_{J}$. In what follows we shall consider $n=0$. Equation (1) is similar to the nonlinear first-order ODE describing the dynamics of the gauge-invariant superconducting phase of a single overdamped JJ with maximum current $I_{J F}$ modulated in field $\left(I_{J F}=\left|\cos \pi \psi_{\mathrm{ex}}\right|\right)$ in which a normalized bias current $i_{B} / 2$ flows. This strict equivalence comes from the hypothesis that the total normalized flux $\psi=\Phi / \Phi_{0}$ linked to the interferometer loop can be taken to be equal to $\psi_{\mathrm{ex}}$. However, being

$$
\psi=\psi_{\mathrm{ex}}+\beta\left(i_{1}-i_{2}\right),
$$


we may say that the above hypothesis may be stated merely by means of the following identity: $\beta=0$. Therefore, for finite values of the parameter $\beta,(1)$ is not anymore valid, and the device behaves as if the equivalent Josephson junction possessed a nonconventional current-phase relation (CPR). In fact, for small finite values of $\beta$, one can see that the following model may be adopted [6]:

$$
\frac{d \phi}{d \tau}+X_{\mathrm{ex}} \sin \phi+\pi \beta Y_{\mathrm{ex}}^{2} \sin 2 \phi=\frac{i_{B}}{2}
$$

where $X_{\mathrm{ex}}=\cos \pi \psi_{\mathrm{ex}}$ and $Y_{\mathrm{ex}}=\sin \pi \psi_{\mathrm{ex}}$. A secondorder harmonic in $\phi$ thus appears in addition to the usual $\sin \phi$ term. The $\sin 2 \phi$ addendum, however, arises solely from electromagnetic coupling between the externally applied flux and the system, as described by (2), when $\beta \neq 0$. Therefore, the non-conventional CPR of the equivalent JJ in the SQUID model cannot be considered as a strict consequence of an intrinsic non-conventional CPR of the single JJs. The Josephson junctions in the device, in fact, could behave in the most classical way, obeying strictly to the Josephson currentphase relation; the interferometer, however, would still show the additional $\sin 2 \phi$ term. The details of the derivation of (3) will be given in the following section for nonstatic (or nonquasistatic) applied fluxes, but a few words on the hypothesis underlying the derivation of (3) are in order in the static case. First of all, the parameter $\beta$ needs to be small, in such a way that only first-order terms are retained in the dynamical equations of the system. Secondly, the quantities $t_{J}=\Phi_{0} / 2 \pi R I_{J}$ and $t_{L}=L / R$, denoting the characteristic time scale of the variables $\phi$ and $\psi$, respectively, are intimately linked to the parameter $\beta$, since $t_{L} / t_{J}=2 \pi \beta \ll 1$. In this way, the flux dynamics for small values of $\beta$ can be considered very fast with respect to the equivalent junction dynamics given in (3). As a consequence, the variable $\phi$ can be assumed to be adiabatic and the equation of motion for $\psi$ in terms of the quasistatic variable $\phi$ can be solved by perturbation analysis. When the information for $\psi$ is substituted back into the dynamical equation for $\phi,(3)$ is finally obtained.

In order to complete the analysis of the reduced model for a d. c. SQUID, in the present work, we consider the dynamics of the superconducting quantum interferometer in the presence of a time-varying applied magnetic flux, whose frequency $\omega$ is considered to be comparable with $t_{L}^{-1}$.

\section{SQUID Dynamics}

If the externally applied magnetic flux does not vary appreciably on the time scale of $t_{L}$, then one can state that (3) still represents the single-junction effective model of a d. c. SQUID. However, when the frequency of the applied flux $\psi_{\text {ex }}$ is comparable with $t_{L}^{-1}$, closer attention to the derivation of the single-junction effective model in the presence of timevarying fields must be paid. Let us thus consider an externally applied flux having d. c. component $A$ and a. c. amplitude $B$, so that

$$
\psi_{\mathrm{ex}}(t)=A+B \sin \omega t
$$

where $\omega \approx t_{L}^{-1}$ is the frequency of the sinusoidal term. A similar analysis, for slowly varying fields and $B \ll A$, has already been carried out in [7]. In the present paper, we make a careful analysis of the problem starting from the SQUID dynamics written in terms of the variables $\phi$ and $\psi$ as follows [7]:

$$
\begin{gathered}
\frac{d \phi}{d \tau}+\cos \pi \psi \sin \phi=\frac{i_{B}}{2}, \\
\pi \frac{d \psi}{d \tau}+\sin \pi \psi \cos \phi=\frac{\psi_{\mathrm{ex}}-\psi}{2 \beta} .
\end{gathered}
$$

We therefore need to consider again all steps in [7], having care to integrate opportunely the right-hand-side term of (5b), in order to obtain a solution for $\psi$ in terms of the superconducting phase by perturbation analysis on $\beta$ for arbitrary values of $A$ and $B$. In this way, the effective dynamics for $\phi$ can be found when the solution for $\psi$ is substituted in the cosine term of (5a). Start by considering the cosine term in (5b) as a quasistatic quantity (i.e., it does not vary appreciably over an interval of time of the order of $\left.t_{L}\right)$. This hypothesis is confirmed by what has already been stated in the previous section that is, while the variables $\phi$ varies on a characteristic time interval $\Delta t_{J}=10^{-10} \mathrm{~s}$, for instance, the variable $\psi$ varies within a time interval $\Delta t_{L}=2 \pi \beta \Delta t_{J} \ll \Delta t_{J}$. Within the former time interval $\Delta t_{J}$, therefore, it is possible to choose a subinterval, of the order of $\Delta t_{L}$, in which the variable $\phi$ does not vary appreciably. We can thus solve (5b), by perturbation analysis, by first setting $\tau=2 \pi \beta \theta$, and by rewriting it as follows:

$$
\frac{d \psi}{d \theta}+2 \beta \sin \pi \psi \cos \phi+\psi(\theta)=\psi_{\mathrm{ex}}(\theta) .
$$

By now setting

$$
\psi(\theta)=\psi_{0}(\theta)+\beta \psi_{1}(\theta)
$$

we find the following two ODEs for $\psi_{0}$ and $\psi_{1}$ :

$$
\frac{d \psi_{0}}{d \theta}+\psi_{0}(\theta)=\psi_{\mathrm{ex}}(\theta)
$$

$$
\frac{d \psi_{1}}{d \theta}+\psi_{1}(\theta)=-2 \cos \phi \sin \pi \psi_{0}
$$

We therefore consider (4) and take the nondecaying solutions of the above system of ordinary differential equations. Recall that an ODE of the type

$$
\frac{d f}{d \theta}+f(\theta)=g(\theta)
$$

has solution $f(\theta)=e^{-\theta} \int^{\theta} g(x) e^{x} \mathrm{~d} x$. Therefore, by considering the non-vanishing solution of (8a) for $\psi_{0}$ at large values of $\theta$, we have

$$
\psi_{0}=A+\frac{B(\sin \tilde{\omega} \theta-\tilde{\omega} \cos \tilde{\omega} \theta)}{1+\widetilde{\omega}^{2}}
$$


where $\widetilde{\omega}=\omega t_{L}$. Having found the solution to $(8 \mathrm{a})$, we can find the solution to ( $8 \mathrm{~b}$ ) by the same type of reasoning. After some rather long calculations, one finds

$$
\psi_{1}=-2 h(\theta) \cos \phi
$$

where

$$
h(\theta)=\sum_{n, k} g_{n, k}\left[\sin \alpha_{n, k}(\theta)-\tilde{\omega} \cos \alpha_{n, k}(\theta)\right],
$$

with $g_{n, k}=J_{n}(\gamma) J_{k}(\gamma \widetilde{\omega}) /\left[1+(n+k)^{2} \widetilde{\omega}^{2}\right], J_{n}(x)$ being the Bessel function of order $n$, and $\alpha_{n, k}=\pi A+(n+k) \tilde{\omega} \theta-k \pi / 2$. The summations in (12) extend from $-\infty$ to $+\infty$, as in other expressions in the present work, if not otherwise stated. Therefore, the SQUID dynamics can be described, to first order in $\beta$, by the following ODE in this case:

$$
\frac{d \phi}{d \tau}+X_{0}(\theta) \sin \phi+\pi \beta h(\theta) Y_{0}(\theta) \sin 2 \phi=\frac{i_{B}}{2},
$$

where $X_{0}(\theta)=\cos \psi_{0}$ and $Y_{0}(\theta)=\sin \psi_{0}$.

\section{Time Averaging the Rapid Variable}

Equation (13) is the differential equation describing the dynamics of a d. c. SQUID in the presence of a time-varying externally applied flux, whose frequency $\omega$ is considered to be comparable with $t_{L}^{-1}$, in such a way that $\widetilde{\omega}=$ $\omega t_{L} \approx 1$. For slowly varying fields $(\widetilde{\omega} \ll 1)$, one can readily verify from $(8 \mathrm{a})$ and $(8 \mathrm{~b})$ that $\psi_{0}(\theta) \rightarrow \psi_{\mathrm{ex}}(\theta)$ and $\psi_{1} \rightarrow-2 \sin \psi_{\mathrm{ex}} \cos \phi$, respectively. In this way, the dynamics described by the quasistatic d. c. SQUID in (3) holds.

For $\tilde{\omega}=\omega t_{L} \approx 1$, time evolution of the two variables, $\phi$ and $\psi$, still occurs with two completely different time scales. Indeed, we have $t_{L}=2 \pi \beta t_{J} \ll t_{J}$. Therefore, flux motion is very fast with respect to the dynamics of the phase variable $\phi$. The only difference, here, is that the externally applied flux $\psi_{\mathrm{ex}}(\theta)$ is able to follow the fast dynamics. Having carefully solved (8a) and (8b), and having found the effective single-junction dynamical equation for a d. c. SQUID, we can determine the effective time-averaged equation, by taking the time average over the fast variable $\psi$. Therefore, we may write

$$
\frac{d \phi}{d \tau}+\left\langle X_{0}(\theta)\right\rangle \sin \phi+\pi \beta\left\langle h(\theta) Y_{0}(\theta)\right\rangle \sin 2 \phi=\frac{i_{B}}{2},
$$

where the symbol $\langle x\rangle$ stands for the time average of the variable $x$. Equation (14) can thus be considered an effective single-junction model for a d. c. SQUID in the presence of a rapidly varying magnetic field $\left(\widetilde{\omega}=\omega t_{L} \approx 1\right)$. The average values $\left\langle X_{0}(\theta)\right\rangle$ and $\left\langle h(\theta) Y_{0}(\theta)\right\rangle$ can be calculated as follows. First of all, set

$$
X_{0}(\theta)=\operatorname{Re}\left\{\exp \left(i \pi \psi_{0}\right)\right\}
$$

Let us next express the exponential of a cosine and a sine terms in $\exp \left(i \pi \psi_{0}\right)$ through the following Bessel function identities:

$$
e^{i a \cos x}=\sum_{n} i^{n} J_{n}(a) e^{i n x}
$$

$$
e^{i a \sin x}=\sum_{n} J_{n}(a) e^{i n x}
$$

In this way, (15) becomes

$$
\left\langle X_{0}(\theta)\right\rangle=\operatorname{Re}\left\{\sum_{n, k} J_{n}(\gamma) J_{k}(\gamma \tilde{\omega})\left\langle e^{i \alpha_{n, k}(\theta)}\right\rangle\right\} .
$$

It is now easy to show that

$$
\left\langle e^{i \alpha_{n, k}(\theta)}\right\rangle=e^{i \pi A} i^{n} \delta_{n,-k},
$$

where the symbol $\delta_{n, m}$ is the Kronecker delta. By inserting (18) in (17), we have

$$
\left\langle X_{0}(\theta)\right\rangle=\cos (\pi A) \rho(\tilde{\omega}, B),
$$

where

$$
\rho(\widetilde{\omega}, B)=J_{0}(\gamma) J_{0}(\gamma \tilde{\omega})+2 \sum_{n}(-1)^{n} J_{2 n}(\gamma) J_{2 n}(\gamma \widetilde{\omega}),
$$

the summation being here carried out from $n=1$ to $+\infty$.

Proceeding in a similar way in finding the effective coefficient of the $\sin 2 \phi$ term, we find

$$
\left\langle h(\theta) Y_{0}(\theta)\right\rangle=\frac{\left(\sum_{n, m \cdot l} g_{n, m} J_{l}(\gamma) J_{n+m-l}(\gamma \tilde{\omega})\right)}{2} .
$$

Having expressed the effective time-averaged terms in (13) in a closed form, we can understand the effect of a high-frequency field on the electrodynamic behaviour, a $\mathrm{d}$. c. SQUID with extremely small value of the parameter $\beta$, for instance. The critical current $i_{C}$ of the device in this case $(\beta=0)$ can be expressed as follows:

$$
i_{C}(\widetilde{\omega}, A, B)=2|\cos \pi A||\rho(\widetilde{\omega}, B)|,
$$

where the quantity $|\rho(\widetilde{\omega}, B)|$ is the extrafactor modifying the usual form of a d. c. SQUID, expressed, in terms of a constant applied flux $A$, as $2|\cos \pi A|$.

This can be seen directly from (13), by setting $\beta=0$ and getting the maximum stationary value for $i_{B}$ with respect to $\phi$.

In Figures 1(a) and 1(b), we thus show the frequency and applied flux behaviour of the factor $|\rho(\widetilde{\omega}, B)|$. In particular, in Figure 1(a), we report the dependence of $i_{C}$ on the amplitude $B$ of the oscillating term in the externally applied flux for various values of the normalized frequency $\widetilde{\omega}$. In Figure 1(b), on the other hand, the critical current is shown as a function of $\widetilde{\omega}$, for various values of $B$. From Figure $1(\mathrm{a})$, we notice Fraunhofer-like patterns of the quantity $|\rho(\widetilde{\omega}, B)|$ with respect to the amplitude $B$. 


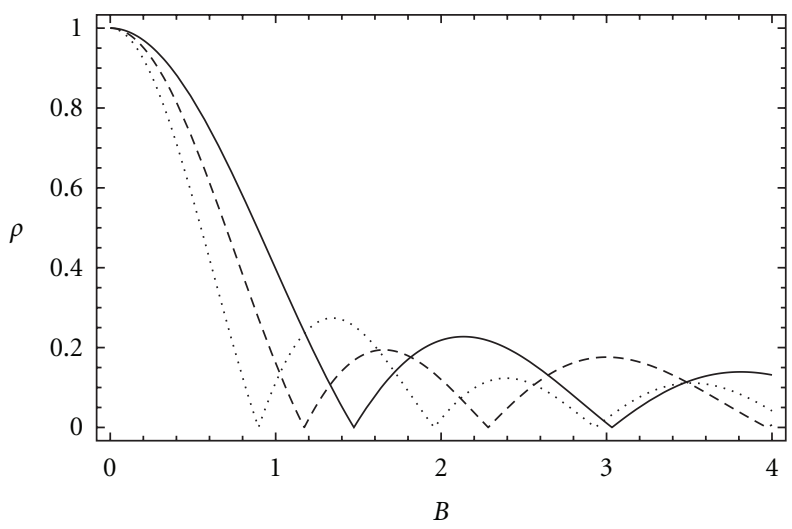

(a)

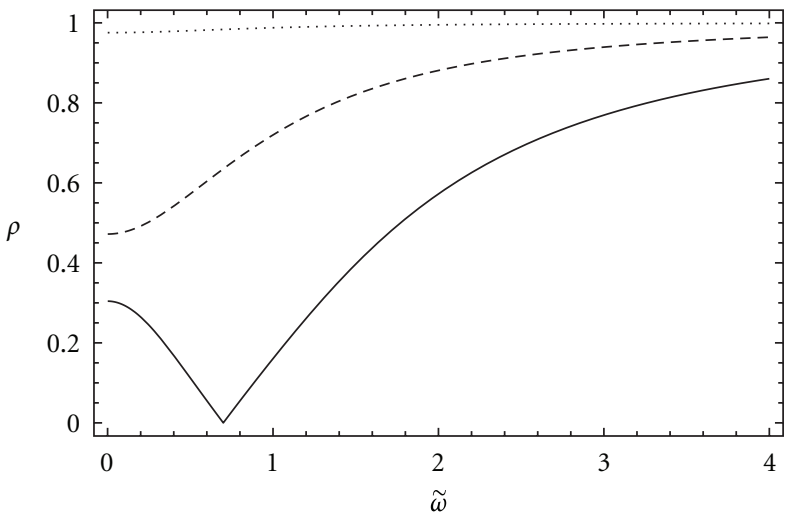

(b)

FIGURE 1: Extra factor $|\rho|$ in the critical current of a d. c. SQUID in the presence of a rapidly varying magnetic flux. In (a), the dependence of $|\rho|$ solely on $B$ is reported, for the following values of $\tilde{\omega}$ : 0.5 (dotted line); 1.0 (dashed line); 1.5 (full line). In (b), the dependence of $|\rho|$ on $\widetilde{\omega}$ is shown for the following values of $B: 0.1$ (dotted line); 0.5 (dashed line); 1.0 (full line).

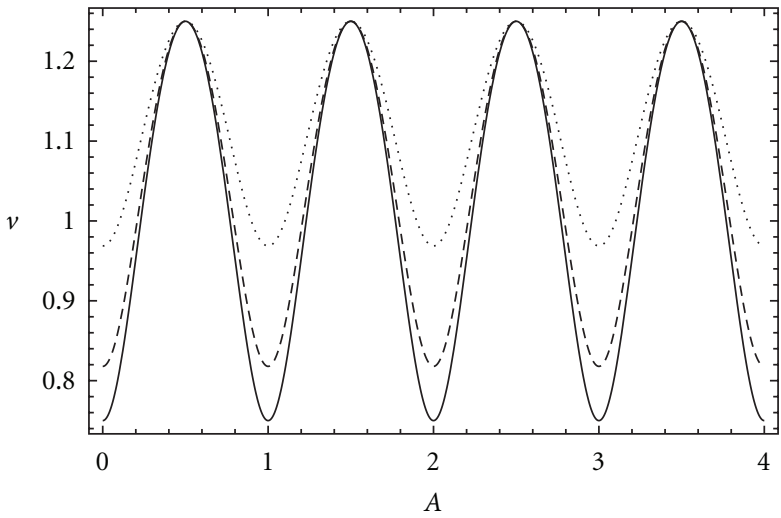

(a)

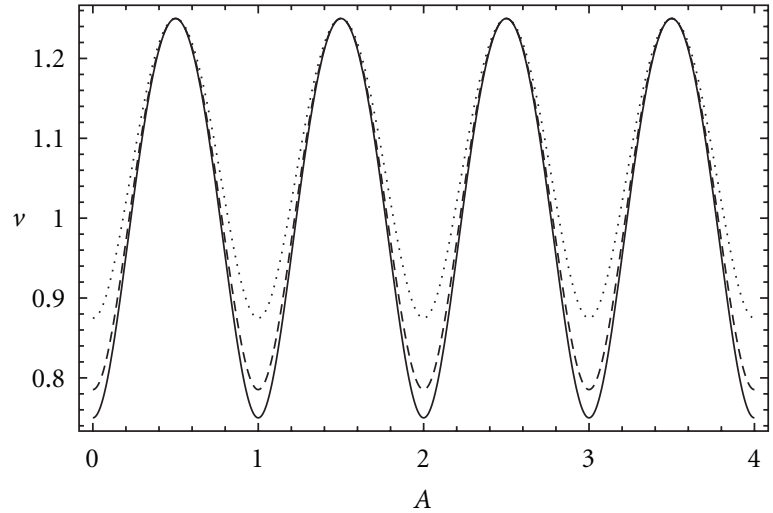

(b)

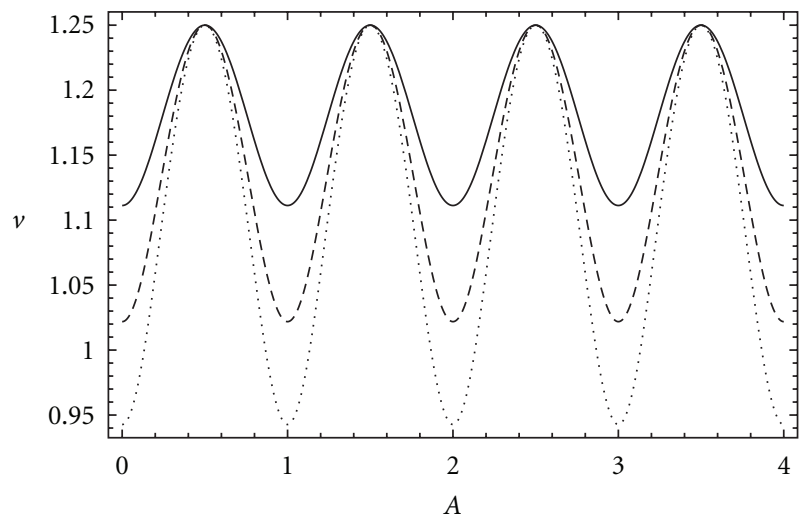

(c)

Figure 2: Voltage versus applied flux $A$ curves for $\beta=0$ and $i_{B}=2.5$. In (a), $\widetilde{\omega}=0.0$, while, $B=0.0$ (full line), $B=0.15$ (dashed line), and $B=0.3$ (dotted line). In (b), $\widetilde{\omega}=1.0$, while $B=0.0$ (full line), $B=0.15$ (dashed line), and $B=0.3$ (dotted line). Finally, in (c), $B=0.5$, while $\tilde{\omega}=0.5$ (full line), $\widetilde{\omega}=1.0$ (dashed line), and $\widetilde{\omega}=1.5$ (dotted line).

The particular shape of these patterns depends on the value of the normalized frequency $\widetilde{\omega}$. When the time-varying portion of the magnetic flux has a fixed amplitude and we let the normalized frequency vary with continuity, the extra factor $|\rho(\widetilde{\omega}, B)|$ behaves as in Figure 1(b). In this respect, we need to further notice that while for small fixed $B$ values the quantity $|\rho(\widetilde{\omega}, B)|$ is always increasing for increasing values of $\widetilde{\omega}$ (see dotted and dashed lines in Figure 1(b)) for values of $B$ approaching 1.0, this character is lost (see full line in Figure 1(b)). 
We can now calculate, for $\beta=0$, the flux-voltage curves $(v$ versus $A$ ) by the following well-known expression [1]:

$$
v=\left\langle\frac{d \phi}{d \tau}\right\rangle=\sqrt{\frac{\left(i_{B}\right)^{2}}{4}-(\cos (\pi A))^{2} \rho^{2}(\widetilde{\omega}, B)}
$$

for $i_{B}>2|\cos (\pi A) \rho(\widetilde{\omega}, B)|$. The important feature in this expression is that these curves depend both on $B$ and $\widetilde{\omega}$ through the extra factor $|\rho(\widetilde{\omega}, B)|$. Because of this dependence, the amplitude of the $v$ versus $A$ curves can be varied, and $B$ and $\tilde{\omega}$ can be viewed as control parameters. In Figures 2(a) $-2(\mathrm{c})$, we thus report the $v$ versus $A$ curves for $i_{B}=$ 2.5 and for various values of $B$ and $\widetilde{\omega}$. In particular, in Figure 2(a), we notice that the amplitude of the $v$ versus $A$ curves obtained at $B=0$ and $\widetilde{\omega}=0$ (full line) decreases as we let $B$ increase to 0.15 first (dashed line) and to 0.30 next (dotted line). The same decreasing behavior is detected in Figure 2(b) for $\widetilde{\omega}=0.1$ when $B$ increases from 0.0 (full line) to 0.15 (dashed line) and to 0.30 (dotted line). In Figure 2(c), finally, by fixing the value of $B$ to 0.5 , we notice that the amplitude of the $v$ versus $A$ curves increases for increasing values of $\widetilde{\omega}$, as shown for $\widetilde{\omega}=0.5$ (full line), $\widetilde{\omega}=1.0$ (dashed line), and $\widetilde{\omega}=1.5$ (dotted line).

A final comment is in order, as far as nonnormalized quantities are concerned, for direct experimental confirmation of the present results. Given that the junction dynamics evolves with characteristic frequencies the order of $1 \mathrm{THz}$, one needs to run the experiment with very rapidly oscillating signals $(10 \mathrm{THz}$ or more) in such a way that normalized frequencies of $\widetilde{\omega}=1.0$ can be obtained.

\section{Conclusion}

By allowing the magnetic flux, applied to a two-junction superconducting quantum interference device, to have an a. c. component in addition to a constant term $A$, we derive the effective reduced single-junction model describing the dynamics of the superconducting phase $\phi$ in the junctions. The single-junction model for the system is obtained by perturbation analysis to first order in the parameter $\beta$. In particular, for $\beta=0$, the critical current of the device is seen to depend on $A$ and on the frequency $\widetilde{\omega}$ and the amplitude $B$ of the a. c. component of the applied magnetic flux in a closed analytic form. From the analysis of the voltage versus applied flux curves it can be argued that the quantities $\widetilde{\omega}$ and $B$ can play the role of additional control parameters in the device. Experimental work confirming the predictions of the present analysis needs to be performed. Further work in extending the present analysis to finite values of $\beta$ is also necessary. Finally, for small a. c. signals, the effect of noise is to be taken in account.

\section{References}

[1] A. Barone and G. Paternò, Physics and Applications of the Josephson Effect, John Wiley \& Sons, New York, NY, USA, 1982.

[2] K. K. Likharev, Dynamics of Josephson Junctions and Circuits, Gordon and Breach, Amsterdam, The Netherlands, 1986.
[3] J. Clarke and A. I. Braginsky, The SQUID Handbook, WileyVCH, Weinheim, Germany, 2004.

[4] M. F. Bocko, A. M. Herr, and M. J. Feldman, "Prospects for quantum coherent computation using superconducting electronics," IEEE Transactions on Applied Superconductivity, vol. 7, no. 2, pp. 3638-3641, 1997.

[5] D. S. Crankshaw and T. P. Orlando, "Inductance effects in the persistent current qubit," IEEE Transactions on Applied Superconductivity, vol. 11, no. 1, pp. 1006-1009, 2001.

[6] F. Romeo and R. De Luca, "Effective non-sinusoidal currentphase dependence in conventional d.c. SQUIDs," Physics Letters A, vol. 328, no. 4-5, pp. 330-334, 2004.

[7] R. De Luca, A. Fedullo, and V. A. Gasanenko, "Pertubation analysis of the dynamical behavior of two-junction interferometers," European Physical Journal B, vol. 58, no. 4, pp. 461-467, 2007. 

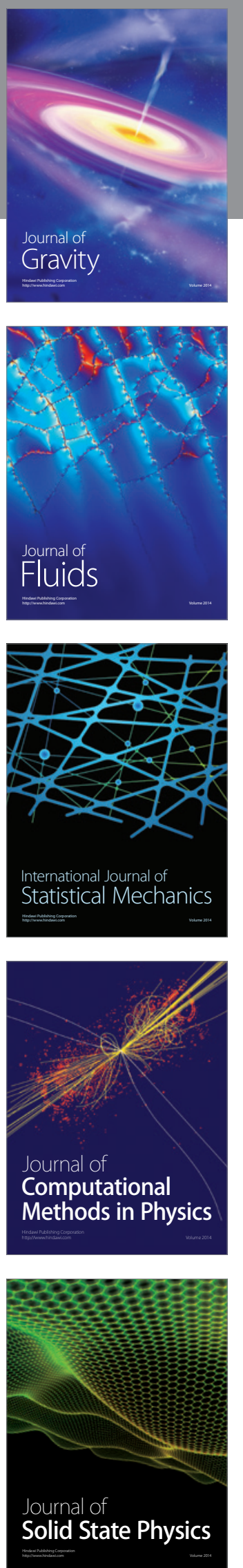
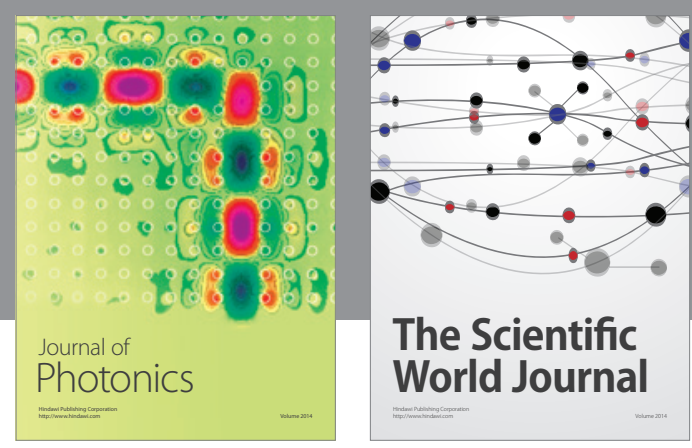

The Scientific World Journal

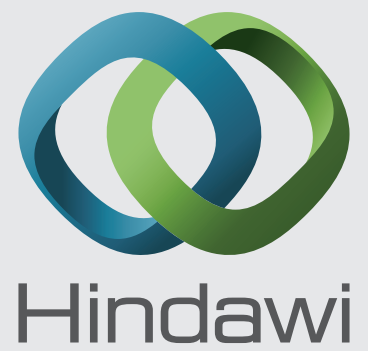

Submit your manuscripts at http://www.hindawi.com
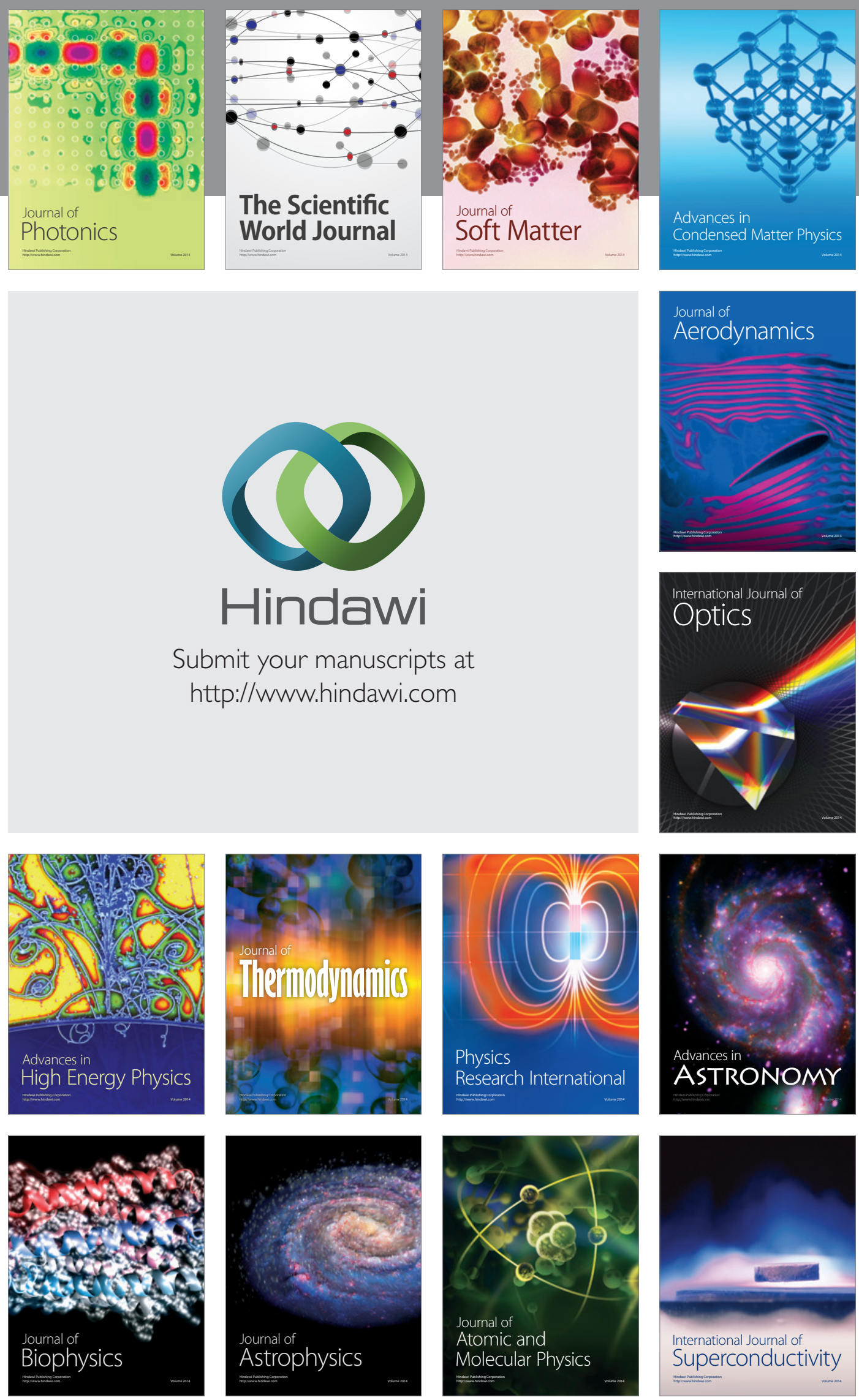
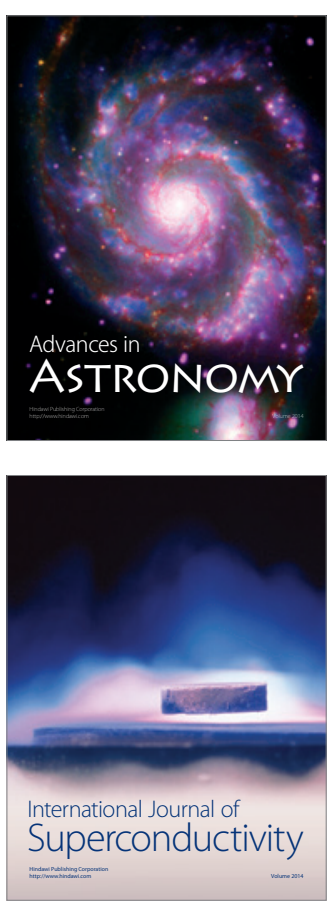